- Practitioners must be aware that the main root canals in a tooth may only provide access to the complexities of the root canal system, which must be fully cleaned of all microorganisms.

- Research has shown that the dental anatomy learned as a dental student may now be out of date.

- Knowledge of canal anatomy is essential in designing and executing access cavities that give straight line access to the main root canals.

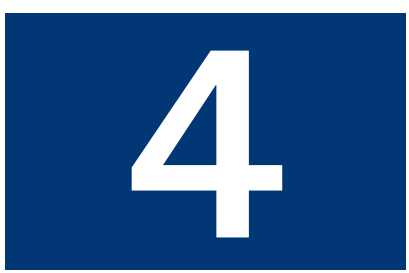

\title{
Endodontics: Part 4 Morphology of the root canal system
}

\author{
P. Carrotte
}

Unless the practitioner is familiar with the morphology of the roots of all teeth, and the associated intricate root canal anatomy, effective debridement and obturation may be impossible. Recent research has improved knowledge and understanding of this intricate aspect of dental practice. After studying this part you should know in what percentage of each tooth type you may expect unusual numbers of root canals and other anatomical variations.

\section{ENDODONTICS}

1. The modern concept of root canal treatment

2. Diagnosis and treatment planning

3. Treatment of endodontic emergencies

4. Morphology of the root canal system

5. Basic instruments and materials for root canal treatment

6. Rubber dam and access cavities

7. Preparing the root canal

8. Filling the root canal system

9. Calcium hydroxide, root resorption, endo-perio lesions

10. Endodontic treatment for children

11. Surgical endodontics

12. Endodontic problems

$1^{*}$ Clinical Lecturer, Department of Adult Dental Care, Glasgow Dental Hospital and School, 378 Sauchiehall Street, Glasgow G2 3JZ

${ }^{*}$ Correspondence to: Peter Carrotte Email:p.carrotte@dental.gla.ac.uk

\section{Refereed Paper}

doi:10.1038/sj.bdj.4811711

(c) British Dental Journal 2004; 197 :

379-383
This part may seem at first sight the most boring in the book, yet it could be the most important in improving clinical practice. Both undergraduate students and dentists on postgraduate courses frequently state that the reason they find root canal treatment so difficult, and the reason surveys frequently report inadequate treatment standards, is because they are working 'blind' Unless a surgical microscope is available it is impossible to see down the root canal - to visualize exactly what the instruments are doing. An understanding of the architecture of the root canal system is therefore an essential prerequisite for successful root canal treatment (see Part 1, Fig. 1). As long ago as 1925, when Hess and Zurcher first published their study, ${ }^{1}$ it became clear that teeth had complicated root canal systems rather than the simplified canals that had been previously described.

Sadly their work, and many similar publications, have largely been overlooked and dentists still remain obsessed with the concept of a 'root canal', a hollow tube down a root which has to be cleaned and shaped, eventually appearing as a nice white line on the post-operative radiograph. Undergraduate students learn the number of canals in each tooth by rote. However, many teeth have more than one canal, as described in this part. Where two canals exist within the same root, for example the mesial root of a lower molar, lateral communication (anastomosis) in the form of fins or accessory canals, occurs between them. Even roots with a single canal will have lateral and accessory canals leaving the main canal. Unless this concept of an entire root canal system is clearly understood, and a method of cleaning and shaping this system employed to address these anastomoses as well as the main canals, infection will remain in the root canal system, and the treatment may fail.

\section{ROOT CANAL SYSTEM}

The pulp chamber in the coronal part of a tooth consists of a single cavity with projections (pulp horns) into the cusps of the tooth (Fig. 1). With age, there is a reduction in the size of the chamber due to the formation of secondary dentine, which can be either physiological or pathological in origin. Reparative or tertiary dentine may be formed as a response to pulpal irritation and is irregular and less uniform in structure.

The entrances (orifices) to the root canals are to be found on the floor of the pulp chamber, usually below the centre of the cusp tips. In cross-section, the canals are ovoid, having their greatest diameter at the orifice or just below it. In longitudinal section, the canals are broader bucco-lingually than in the mesiodistal plane. The canals taper towards the apex, following the external outline of the root. The narrowest part of the canal is to be found at the 'apical constriction', which then opens out as the apical foramen and exits to one side between 0.5 and $1.0 \mathrm{~mm}$ from the anatomical apex. Deposition of secondary cementum may place the apical foramen as much as $2.0 \mathrm{~mm}$ from the anatomical apex. It must be realized, however, that the concept of a 'single' root canal with a 'single' apical foramen is mistaken. The root canal may end in a delta of small canals, and during root canal treatment cleaning techniques should be employed to address this clinical situation.

\section{LATERAL AND ACCESSORY CANALS}

As previously discussed, lateral canals form channels of communication between the main body of the root canal and the periodontal ligament space. They arise anywhere along its length, at right angles to the main canal. The term 'accessory' is usually reserved for the small 
Fig. 1 The pulp chamber in the coronal part of the tooth consists of a single cavity with projections (pulp horns) into the cusps of the tooth.
Fig. 3 The basic pulp canal shape and suggested access cavity openings in the maxilliary teeth.

Fig. 4 The basic pulp canal shape and suggested access cavity openings in the mandibular teeth
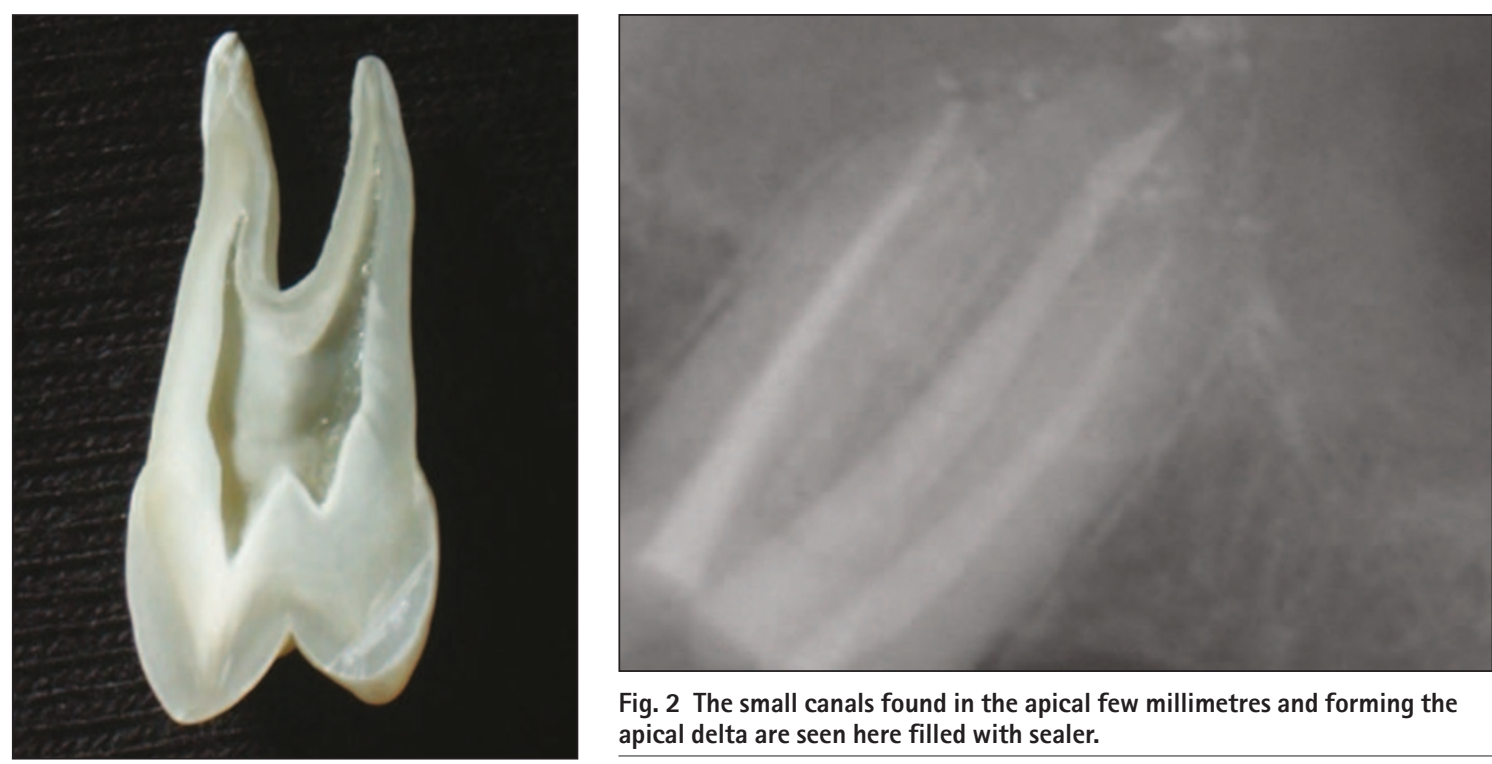

Fig. 2 The small canals found in the apical few millimetres and forming the apical delta are seen here filled with sealer. canals found in the apical few millimetres and forming the apical delta (Fig. 2). Both lateral and accessory canals develop due to a break in 'Hertwig's epithelial root sheath' or, during development, the sheath grows around existing blood vessels. Their significance lies in their relatively high prevalence, Kasahara et al. ${ }^{2}$ finding $60 \%$ of central incisors with accessory canals, and 45\% with apical foramina distant from the actual tooth apex. Kramer found that the diameter of some lateral canals was often wider than the apical constriction. ${ }^{3}$ Lateral canals are
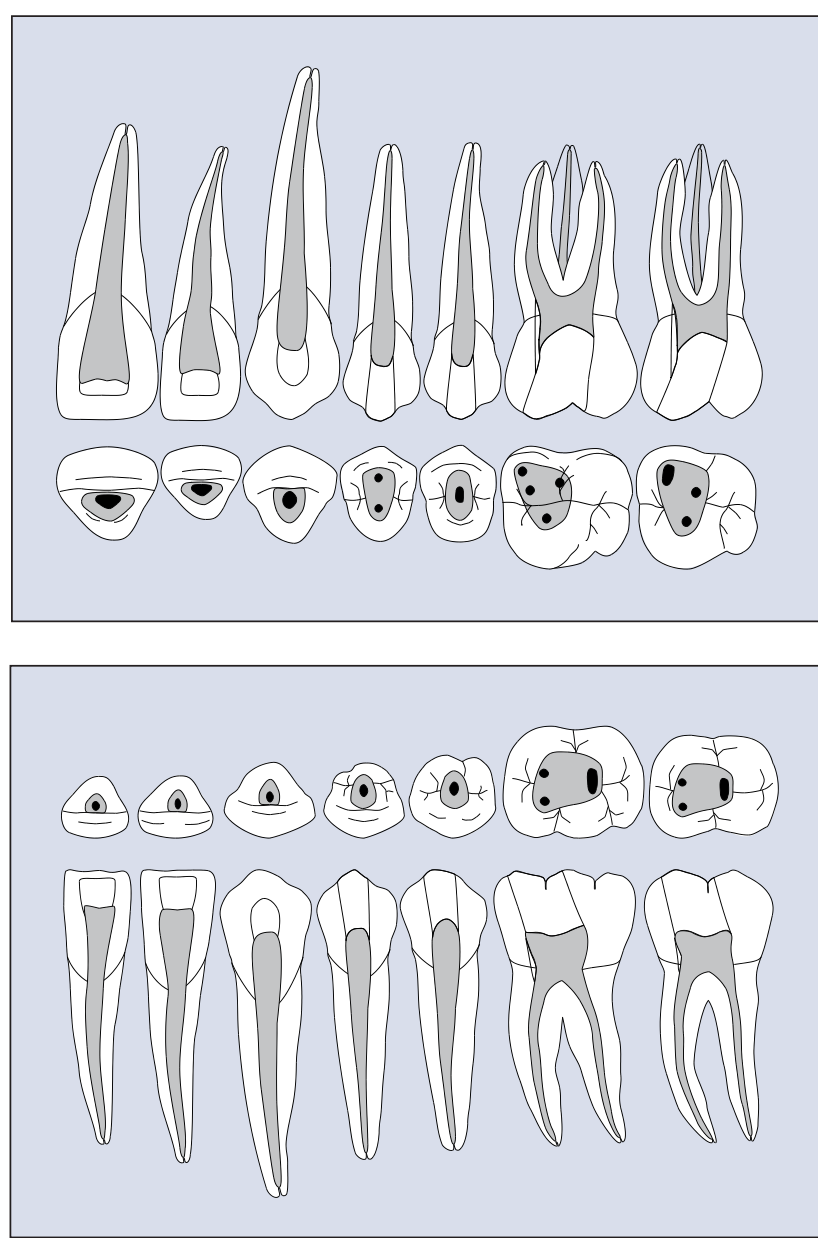

impossible to instrument and can only be cleaned by effective irrigation with a suitable antimicrobial solution. Consequently, sealing such canals is only moderately successful.

The following descriptions of normal canal morphology and access cavities are illustrated in Figure 3 (maxilliary) and Figure 4 (mandibular).

\section{MAXILLARY CENTRAL INCISORS}

These teeth almost always have one canal. When viewed on radiographs the canal appears to be fairly straight and tapering, but labiopalatally the canal will tend to curve either towards the labial or palatal aspect at about the apical third level. One feature to note is the slight narrowing of the lumen at the cervical level, which immediately opens up into the main body of the canal. The inverted-triangular shaped access cavity is cut with its base at the cingulum to give straight line access.

\section{MAXILLARY LATERAL INCISOR}

Similar in shape to the central incisors, but fractionally shorter, the apical third tends to curve distally and the canal is often very fine with thin walls. Labiopalatally, the canal is similar to the central incisor, but there is often a narrowing of the canal at the apical third level. The root is more palatally placed, an important point when any periradicular surgical procedures are carried out on this tooth. The access cavity is similar to the central incisor.

\section{MAXILLARY CANINE}

As well as being the longest tooth in the mouth, its oval canal often seems very spacious during instrumentation. However, there is usually a sudden narrowing at the apical $2-3 \mathrm{~mm}$; this leads to a danger of overinstrumentation if too large a file is used at this level. The length of this tooth can be difficult to determine on radiographs, as the apex tends to curve labially and the tooth will appear to be shorter than it actually is. The oval shape of the root canal is reflected in the shape of the access cavity. 


\section{MAXILLARY FIRST PREMOLAR}

Typically, this tooth has two roots with two canals. In many ways this is the most difficult tooth to treat, as it can have a complex canal system. Variations range from one to three roots, (Fig. 5), but there are nearly always at least two canals present, even if they exit through a common apical foramen. The roots of these teeth are very delicate and at the apical third they may curve quite sharply buccally, palatally, mesially or distally, so instrumentation needs to be carried out with great care (Fig. 6). In a small percentage of cases the buccal root may subdivide into two canals in the apical third, as shown in Figure 7. An oval access cavity is cut between the cusp tips, being wider buccopalatally than mesiodistally.

\section{MAXILLARY SECOND PREMOLAR}

In $40 \%$ of cases, this tooth, which is similar in length to the first premolar, has one root with a single canal. Two canals may be found in about $58 \%$ of cases. ${ }^{4}$ The configuration of the two canals may vary with two separate canals and two exits, two canals and one common exit, one canal dividing and having two exits. In one study, ${ }^{5}$ it was found that 59\% of maxillary second premolars had accessory canals. As with the first maxillary premolar, the apical third of the root may curve quite considerably, mainly to the distal, sometimes buccally. The access cavity is similar to the first premolar.

\section{MAXILLARY FIRST MOLAR}

This tooth has three roots. The palatal root is the longest, with an average length of $22 \mathrm{~mm}$; the mesiobuccal and distobuccal roots are slightly shorter, at $21 \mathrm{~mm}$ average length. The percentage of mesiobuccal roots having two canals reported in the literature has increased steadily as research techniques have developed. In vitro studies have usually reported a higher incidence than in vivo studies. Stropko, reporting an extensive in vivo study, ${ }^{6}$ found second canals in $73 \%$ of cases before the use of an operating microscope, but 93\% following its use. The canals of the mesiobuccal root are often very fine and difficult to negotiate; consequently, more errors in instrumentation occur in this tooth than in almost any other. Anastomosis between these two canals may take the form of narrow canals or wide fins, both almost impossible to instrument. The curvature of the roots can be difficult to visualize from radiographs, and the second mesiobuccal canal is nearly always superimposed on the primary mesiobuccal canal. The palatal root has a tendency to curve towards the buccal and the apparent length on a radiograph will be shorter than its actual length. The access cavity represents the shape of the pulp chamber, enlarged slightly, and flared up on to the mesiobuccal aspect of the occlusal surface to accommodate the angle of instrument approach when working at the back of the mouth.

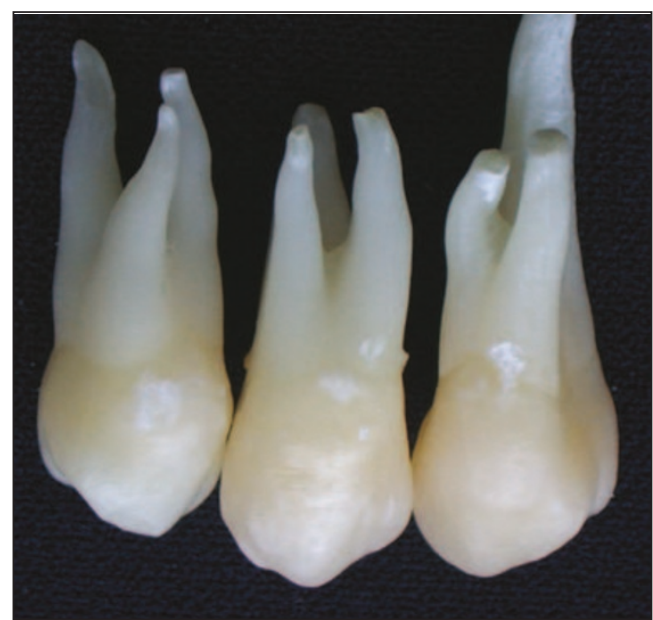

Fig. 5 Examples of upper premolars with three roots.

\section{MAXILLARY SECOND MOLAR}

This tooth is similar to the first maxillary molar, but slightly smaller and shorter, with straighter roots and thinner walls. Usually there are only three canals and the roots are sometimes fused. The access cavity is the same as for the first molar, modified further to accommodate the angle of approach.

\section{MAXILLARY THIRD MOLAR}

The morphology of this tooth can vary considerably, ranging from a copy of the first or second maxillary molar to a canal system that is quite complex. They are best explored with a wide access cavity and direct vision of the individual canal anatomy.
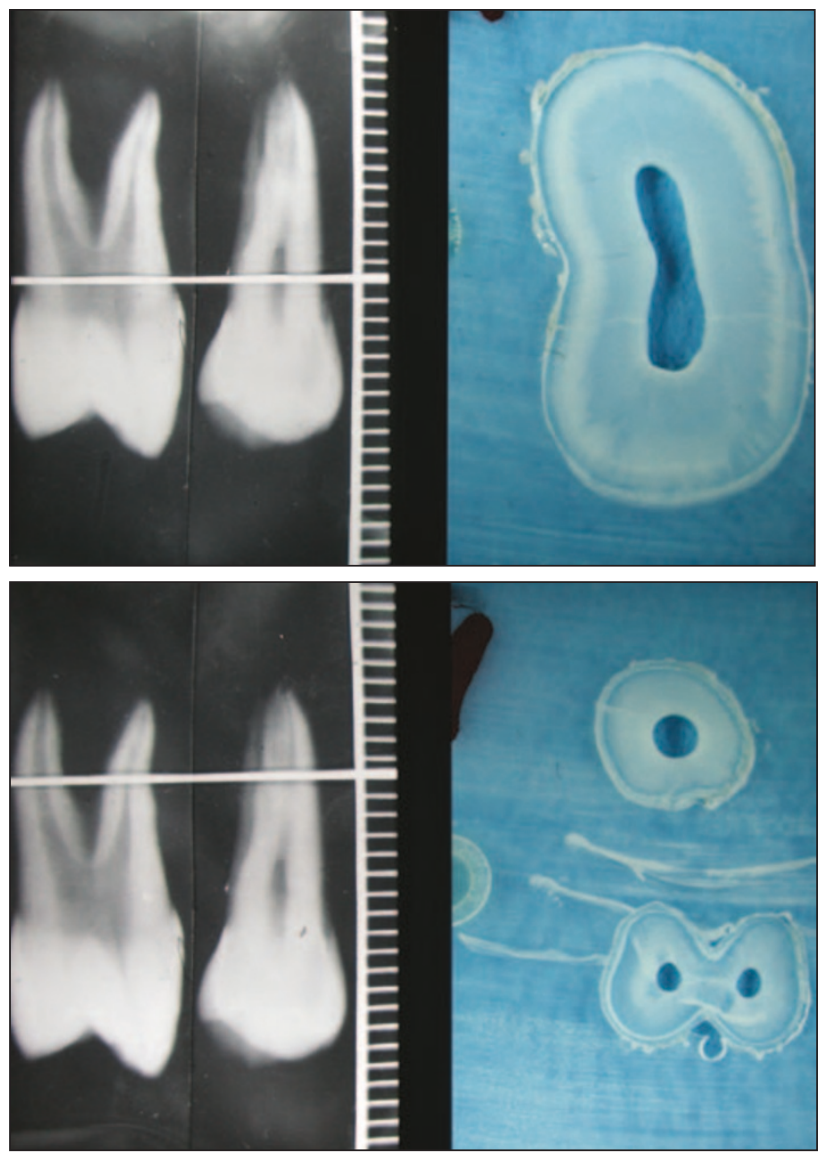

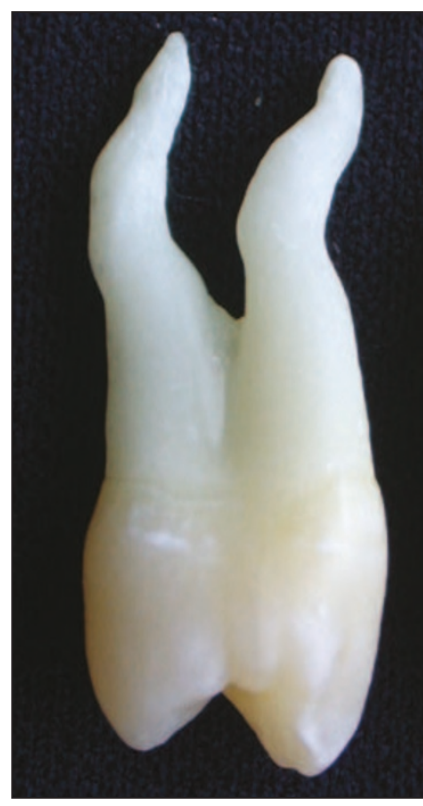

Fig. 6 The roots of upper first premolars are very delicate and may curve quite sharply buccally, palatally, mesially or distally, so instrumentation needs to be carried out with great care. 
Fig. 8 Lower canines may occasionally be found with two separate roots.
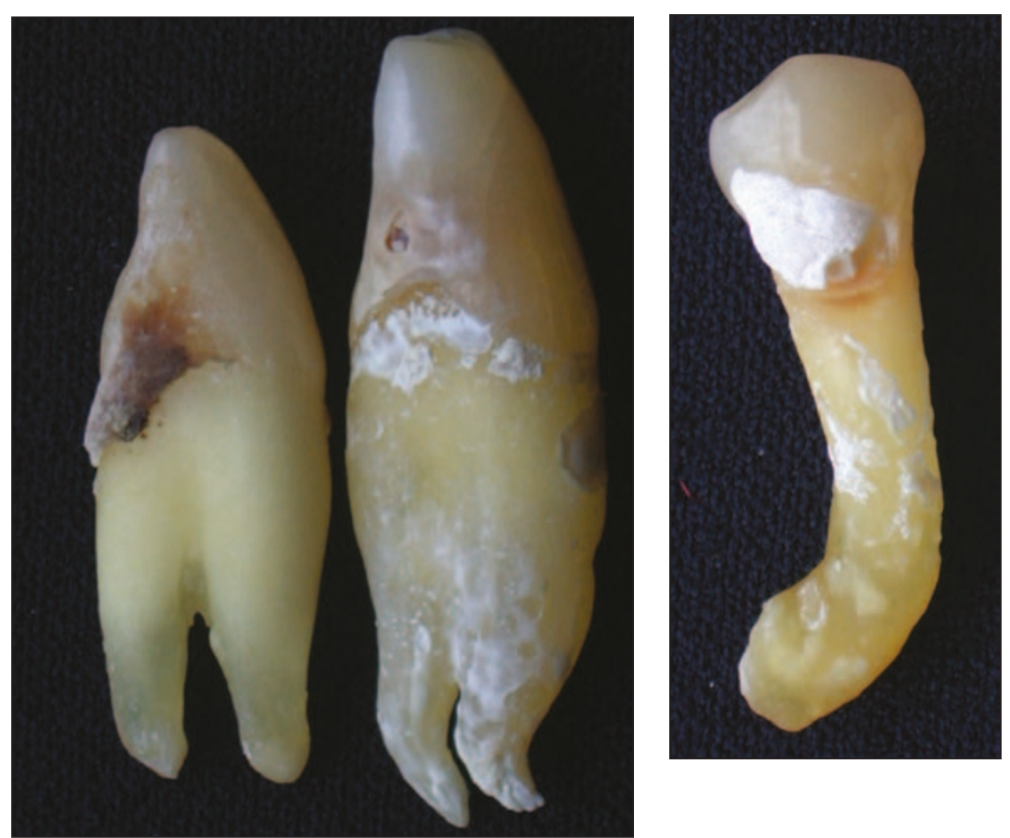

Fig. 9 A lower second premolar with a severe distal curve at the apex.
Table 1 Average root canal configurations

\begin{tabular}{|c|c|c|c|}
\hline Tooth & Average Length & No. of roots & No. of canals \\
\hline \multicolumn{4}{|l|}{ Maxillary anteriors } \\
\hline Central incisor & $22.5 \mathrm{~mm}$ & 1 & 1 \\
\hline Lateral incisor & $22.0 \mathrm{~mm}$ & 1 & 1 \\
\hline Canine & $26.5 \mathrm{~mm}$ & 1 & 1 \\
\hline \multicolumn{4}{|l|}{ Maxillary premolar } \\
\hline \multirow[t]{3}{*}{ First premolar } & $20.6 \mathrm{~mm}$ & $2-3$ & $1(6 . \%)$ \\
\hline & & & $2(95 \%)$ \\
\hline & & & $3(1 \%)$ \\
\hline \multirow[t]{3}{*}{ Second premolar } & $21.5 \mathrm{~mm}$ & $1-3$ & $1(75 \%)$ \\
\hline & & & $2(24 \%)$ \\
\hline & & & $3(1 \%)$ \\
\hline \multicolumn{4}{|l|}{ Maxillary molars } \\
\hline \multirow[t]{2}{*}{ First molar } & $20.8 \mathrm{~mm}$ & 3 & $4(93 \%)$ \\
\hline & & & $3(7 \%)$ \\
\hline \multirow[t]{2}{*}{ Second molar } & $20.0 \mathrm{~mm}$ & 3 & $4(37 \%)$ \\
\hline & & & $3(63 \%)$ \\
\hline Third molar & $17.0 \mathrm{~mm}$ & $1-3$ & \\
\hline \multicolumn{4}{|c|}{ Mandibular anteriors } \\
\hline \multirow[t]{2}{*}{ Central incisor } & $20.7 \mathrm{~mm}$ & 1 & $1(58 \%)$ \\
\hline & & & $2(42 \%)$ \\
\hline \multirow[t]{2}{*}{ Lateral incisor } & $20.7 \mathrm{~mm}$ & $1-2$ & $1(58 \%)$ \\
\hline & & & $2(42 \%)$ \\
\hline \multirow[t]{2}{*}{ Canine } & $25.6 \mathrm{~mm}$ & 1 & $1(94 \%)$ \\
\hline & & & $2(6 \%)$ \\
\hline \multicolumn{4}{|c|}{ Mandibular premolars } \\
\hline \multirow[t]{2}{*}{ First premolar } & $21.6 \mathrm{~mm}$ & 1 & $1(73 \%)$ \\
\hline & & & $2(27 \%)$ \\
\hline \multirow[t]{2}{*}{ Second premolar } & $22.3 \mathrm{~mm}$ & 1 & $1(85 \%)$ \\
\hline & & & $2(15 \%)$ \\
\hline \multicolumn{4}{|l|}{ Mandibular molars } \\
\hline \multirow[t]{2}{*}{ First molar } & $21.0 \mathrm{~mm}$ & $2-3$ & $3(67 \%)$ \\
\hline & & & $4(33 \%)$ \\
\hline \multirow[t]{3}{*}{ Second molar } & $19.8 \mathrm{~mm}$ & 2 & $2(13 \%)$ \\
\hline & & & $3(79 \%)$ \\
\hline & & & $4(8 \%)$ \\
\hline Third molar & $18.5 \mathrm{~mm}$ & $1-2$ & \\
\hline
\end{tabular}

\section{MANDIBULAR CENTRAL AND LATERAL} INCISORS

The morphology of these two teeth is very similar. The central incisor has an average length of $20.5 \mathrm{~mm}$ and the lateral is a little longer with an average length of $21 \mathrm{~mm}$. Over $40 \%$ of these teeth have two canals, but only just over 1\% have two separate foramina. Careful reading of the pre-operative radiograph may show a change in the radiodensity of the root canal, indicating division into two separate canals, and a correctly designed access cavity will facilitate checking for a second canal. This is oval in shape, commencing above the cingulum and almost notching the lingual incisal edge.

\section{MANDIBULAR CANINE}

This tooth is similar to its opposite number, although not as long. On rare occasions, two roots may exist and this can cause difficulty with instrumentation (Fig. 8). An oval access cavity is again indicated.

\section{MANDIBULAR FIRST PREMOLAR}

The canal configuration of this tooth can be quite complex. Vertucci ${ }^{7}$ has shown that the single canal normally found may divide into two canals and two apical foramina in $25 \%$ of cases. It is the way in which the second canal branches that can cause difficulty with instrumentation. Occasionally, the canal terminates with an extensive delta, making obturation of the accessory canals even more challenging. As in the upper premolars, the access cavity is oval between the cusp tips.

\section{MANDIBULAR SECOND PREMOLAR}

This tooth is similar to the first premolar, except that the incidence of a second canal is very much lower. One study stated this to be $12 \%{ }^{7}$ Another study revealed that only $2.5 \%$ had two 


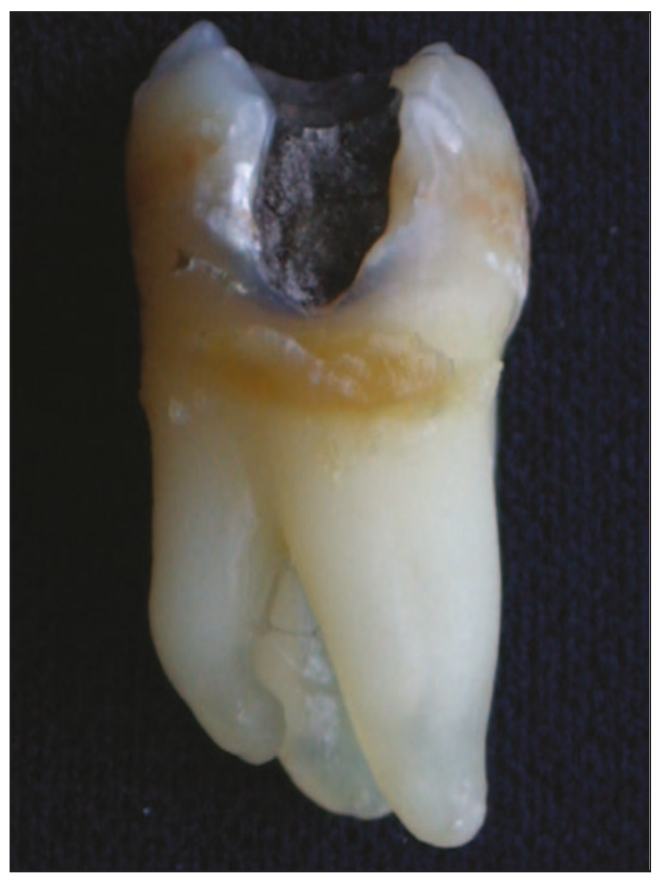

Fig. 10 Lower first molars may occasionally be found with two separate distal roots.

apical foramina. ${ }^{8}$ Consequently, it is a much easier tooth to treat compared with the mandibular first premolar, unless the radiograph reveals a sharp distal curve at the apex as shown in the extracted tooth at Figure 9.

\section{MANDIBULAR FIRST MOLAR}

This is often the most heavily restored tooth in the adult dentition and seems to be a frequent candidate for root canal treatment. Generally there are two roots and three canals: two canals in the mesial root and one large oval canal distally. According to Skidmore and Bjorndal, ${ }^{9}$ one third of these molars have four canals. Occasionally, three roots are to be found: usually two distal and one mesial (Fig. 10), rarely one distal and two mesial. Anastomoses occur between the canals and accessory communication with the furcation area is a frequent finding. The mesiobuccal canal tends to exhibit the greatest degree of curvature. The access cavity once again represents the shape of the pulp chamber, enlarged slightly, and flared up on to the mesiobuccal aspect of the occlusal surface to accommodate the angle of instrument approach when working at the back of the mouth.

\section{MANDIBULAR SECOND MOLAR}

This tooth is similar to the mandibular first molar, although a little more compact. The mesial canals tend to lie much closer together, and the incidence of two canals distally is much less. This tooth seems to be more susceptible to vertical fracture. Occasionally, the root canals may join in a buccal fin giving a 'C-shaped' canal, which may lead all the way to the apex. ${ }^{10}$ The access cavity is similar to that of the first molar.

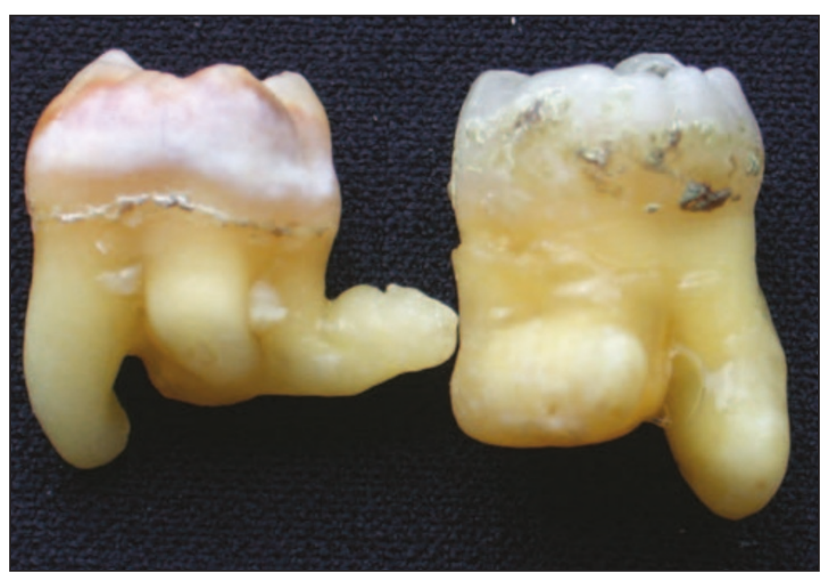

Fig. 11 The developmental anatomy of lower third molars may be quite bizarre.

\section{MANDIBULAR THIRD MOLAR}

Together with the maxillary third molar, this tooth displays some of the most irregular canal configurations to be found in the adult dentition, as seen in Figure 11. However, the mesial inclination of the tooth generally makes access easier. The canal orifices are not too difficult to locate, but the degree of curvature of the apical half of the root canal system is often pronounced. Added to this, the apex is frequently poorly developed and lies close to the inferior alveolar canal. A large access cavity allowing direct visualization of the floor of the pulp chamber enables the canal orifices to be identified.

It should always be remembered that whilst the above descriptions are the norm, occasionally other teeth may be encountered with unusual or even bizarre anatomy. This may only be discovered after the treatment has failed and the tooth has been extracted. Clues may sometimes be found by careful examination of the radiographs, especially with the use of magnification, as described in Part 5.

1. Hess W, Zurcher E. The anatomy of the root canals of the teeth of the permanent dentition and the anatomy of the root canals of the deciduous dentition and the first permanent molars. London: Basle, Sons and Danielson, 1925.

2. Kasahara E,Yasuda E, Yamamoto A, Anzai M. Root canal systems of the maxillary central incisor. J Endod 1990; 16: 158-161.

3. Kramer I R. The vascular architecture of the human dental pulp. Arch Oral Biol 1960; 2: 177-189.

4. Bellizi R, Hartwell G. Radiographic evaluation of root canal anatomy of in vivo endodontically treated maxillary premolars. J Endod 1985; 11: 37-39.

5. Vertucci F J, Seeling A, Gillis R. Root canal morphology of the human maxillary second premolar. Oral Surg 1974; 38: 456-464.

6. Stropko J J. Canal morphology of maxillary molars: clinical observations of canal configurations. J Endod 1999; 25: 446-450.

7. Zillich R, Dowson J. Root canal morphology of the mandibular first and second premolars. Oral Surg 1973; 36: 738-744.

8. Vertucci F J. Root canal morphology of mandibular premolars. J Am Dent Assoc 1978; 97: 47-50.

9. Skidmore A E, Bjorndal A M. Root canal morphology of the human mandibular first molar. Oral Surg 1971; 32: 778-784.

10. Melton D C, Krall K V, Fuller M W. Anatomical and histological features of C-shaped canals in mandibula second molars. J Endod 1991; 17: 384-388. 